\title{
The complexity of pelvic floor dysfunction: Translating evidence into practice. A physical therapist's perspective on an integrated approach
}

\author{
Corlia Brandt* and van Vuuren ECJ \\ Department of Physiotherapy, Faculty of Health Sciences, Wits, South Africa
}

Pelvic floor dysfunction (PFD) is an under-investigated condition with a complex, multifactorial pathophysiology. Approximately 40$60 \%$ of women between the ages of 50 and 60 years may present with PFD [1]. Typical symptoms include urinary or faecal incontinence, pelvic pain and sexual dysfunction which may be present for years, leading to adaptation of lifestyle and physical activities [1]. However, very little is known about the movement impairments and activity restrictions in women with PFD underlying these adaptations [2,3]. This poses a current challenge to effective conservative management of PFD with a consequent negative effect on global costing [4].

Although the signature pedagogy of physiotherapy is defined as human movement [5], a recent clinical commentary stated that there is not a system in place that guides the diagnosis and treatment of PFD based on movement impairments. The proposal is that diagnosis and treatment of patients with PFD should be guided by a combination of movement-related symptoms and signs, impairments, activity and participation restrictions (International Classification of Function, Disability and Health [ICF] framework). The movement impairment should however be the guiding factor on which the conservative management of these patients is based $[2,3]$.

The controversy in research regarding the biomechanical behaviour of the pelvic floor muscles in relation to other muscle function (movement impairment), together with the dynamic interaction between the impairment, functioning and disability, the health condition (namely PFD) and contextual factors, leads to complicated clinical problem solving. Contextual factors include both personal and environmental factors [6]. Several studies have indicated that contextual factors, such as older age, menopause, body mass index (BMI), birth history and a history of previous surgery, are associated with PFD [7]. Many of the risk factors for PFD are also risk factors for the development of lifestyle diseases, such as hypertension and cardiovascular disease. These risk factors include pregnancy and childbirth, deficient connective tissue (varicose veins, hernia, haemorrhoids), hormonal factors, poor diet, smoking, obesity, lack of exercise, ageing and menopause $[8,9]$. It could be postulated that the latter diseases may therefore also present in patients with PFD, adding to their decreased QoL and lifestyle changes [9]. This indicates the complexity of PFD and the need for inter-disciplinary management approaches.

Translation of evidence into clinical practice is complicated by the fact that the context and environment (and therefore the interactions between the different factors) differ in different populations, health care settings and geographical areas. It is to be expected that research findings on movement impairments, activity limitations and participation restrictions may not always be generalisable to different populations, due to the differences and interactions between these factors. Population-specific studies may contribute to explain some complexities and clarify the current lack of understanding regarding PFD [10,11].

Population-specific studies should lead to a conceptual framework of information on personal health care, prevention and promotion by indicating social hindrances in different populations, who need support and facilitation. Secondly, the studies may identify biopsychosocial aspects that may affect movement impairments, and the interactions with the identified disability, activity limitations and participation restrictions. Lastly, they may contribute to the evaluation and policy formulation regarding women's and pelvic health in a country/population, and therefore development of the current health care system [6].

Translating population specific evidence into effective service delivery and higher education in women's and pelvic health, will contribute to the excellence and innovation in the field. This should be driven by interactions between population specific research, the different healthcare sectors, education and clinical practice; including input from a patient perspective to inter-disciplinary specialist opinions, and shared leadership [5].

Protocols should be developed to determine what works, in which setting, for whom, in what circumstances, and why; by determining a theory to explain how context may influence service delivery and clinical outcomes (C-M-O configuration) [12]. The aim should be to inform an inter-disciplinary service delivery models to address effective management of PFD in different countries, considering differences in healthcare facilities; cultural beliefs; higher education institutions to provide training and research; resources; staff, student and patient profiles and skills. Evidence should be translated into practice, and practice into evidence.

${ }^{\star}$ Correspondence to: Corlia Brandt, Department of Physiotherapy, Faculty of Health Sciences, Private Bag 3, Wits, 2050, South Africa, Tel: +27 117172014 Fax: 2786663 5776; E-mail: Corlia.Brandt@wits.ac.za

Received: September 29, 2019; Accepted: October 09, 2019; Published: October 14,2019 


\section{References}

1. Wang YC, Hart DL, Mioduski JE (2012) Characteristics of patients seeking outpatient rehabilitation for pelvic-floor dysfunction. Phys Ther 92: 1160-1174. [Crossref]

2. Brandt C, Janse van Vuuren EC (2019) An International Classification of Function, Disability and Health (ICF)-based investigation of movement impairment in women with pelvic organ prolapse. S Afr J Physiother [Crossref]

3. Spitznagle T, Cabelka C, Clinton S, Abraham K, Norton B (2017) Diagnosis dialog for women's health conditions: The process and proposed pelvic floor muscle diagnoses, Section on Women's Health. American Physical Therapy Association 41: 154-162.

4. Milsom I (2016) Prediction and prevention of pelvic floor dysfunction. ANZCJ 22: 87.

5. Jensen GM, Hack LM, Nordstrom T, Gwyer J, Mostrom E (2017) National study of excellence and innovation in physical therapist education: Part $2-\mathrm{A}$ call to reform. Phys Ther 97: 875-888. [Crossref]

6. World Health Organisation (2002) International classification of functioning, disability and health, Geneva.
7. Weber AM, Richter HE (2005) Pelvic organ prolapse. Obstet Gynecol 106: 615-632 [Crossref]

8. Bo K, Berghmans B, Morkved S, Van Kampen M (2015) Physical therapy for the pelvic floor. Bridging science and clinical practice, 2nd edn., Churchill Livingstone, Edinburgh.

9. Leuzzi C, Modena MG (2011) Hypertension in postmenopausal women. Pathophysiology and treatment. High Blood Press Cardiovasc Prev 18: 13-18. [Crossref]

10. Brandt C, Janse van Vuuren EC (2019) Dysfunction, activity limitations, participation restriction and contextual factors in South African women with pelvic organ prolapse. S Afr J Physiother 75: 933. [Crossref]

11. Srikrishna S, Robinson D, Cardozo L, Gonzalez J (2008) Is there a difference in patient and physician quality of life evaluation in pelvic organ prolapse. Int Urogynecol $J$ Pelvic Floor Dysfunct 19: 517-520. [Crossref]

12. Mirzoev T, Etiaba E, Ebenso B, Uzochukwu B, Manzano A, et al. (2016) Study protocol: realist evaluation of effectiveness and sustainability of a community health workers programme in improving maternal and child health in Nigeria. Implement $S c i$ 11: 83. [Crossref]

Copyright: (C2019 Brandt C. This is an open-access article distributed under the terms of the Creative Commons Attribution License, which permits unrestricted use, distribution, and reproduction in any medium, provided the original author and source are credited. 